Vietnam Journal of Mechanics, VAST, Vol.39, No. 1 (2017), pp. 79 -95

DOI:10.15625/0866-7136/9342

\title{
A NUMERICAL STUDY OF ULTRASONIC RESPONSE OF RANDOM CORTICAL BONE PLATES
}

\author{
A. Abdoulatuf, V-H. Nguyen*, C. Desceliers, S. Naili \\ University of Paris-Est, France \\ *E-mail: vu-hieu.nguyen@univ-pars-est.fr \\ Received September 15, 2016
}

\begin{abstract}
A probabilistic study on ultrasound wave reflection and transmission from cortical bone plates is proposed. The cortical bone is modeled by an anisotropic and heterogeneous elastic plate sandwiched between two fluids and has randomly varied elastic properties in the thickness direction. A parametric stochastic model is proposed to describe the elastic heterogeneity in the plate. Reflection and transmission coefficients are computed via the semi-analytical finite element (SAFE) method. The effect of material heterogeneity on reflected and transmitted waves is investigated from a probabilistic point of view. The parametric study highlights effects of the uncertainty of material properties on the reflection and transmission coefficients by varying the frequency, angle of incidence and bone thickness.
\end{abstract}

Keywords: Ultrasound, cortical bone, transmission/reflection coefficients, semi-analytical finite element method, random material properties.

\section{INTRODUCTION}

In recent years, the development of quantitative ultrasound methods (QUS) to asses mechanical behaviors of bone tissue is a key issue [1-3]. It has been shown that speed of sound (SOS) and broadband ultrasound attenuation (BUA) provided valuable information about bone structure in clinical assessment [4]. Ultrasound attenuation in bones is due to scattering and absorption effects, which may reflect the deterioration of the bone structure [3-5].

For studying long bones such as femur and tibia, ultrasonic measurements using an axial transmission technique, which has been initiated by initially by Lowet and Van der Perre [6], have been developed. This technique consists of use a set of ultrasonic transmitters and receivers placed on a line in contact with the skin along the bone axial axis. The analysis of the signals received can allow to estimate velocities of different kinds of wave propagating in the bone structure and thus to quantify some mechanical properties of bones (such as density, rigidity and thickness) [7-11]. The attenuation of

(C) 2017 Vietnam Academy of Science and Technology 
cortical bone may also been evaluated by analyzing the reflected wave from bone surface [12].

In this context, the present work focuses on the study of the reflection and transmission ulatrasonic waves from cortical bones plates. Basically, ultrasonic testings of long bones may be modeled by using a coupled system which consists of a constant-thickness plate-like or cylindrical-like solid (for representing the cortical bone) sandwiched between two fluids (for representing soft tissues and marrow). It is has been shown that the porosity in the radial direction (which is defined in the bone's cross-section plan) is heterogeneous $[13,14]$. As the macroscopic mechanical properties of bones strongly depend on its porosity $[15,16]$, cortical bone would naturally be considered as a functionally graded material. Thus the model employed in this study involves an anisotropic elastic plate sandwiched between two homogeneous fluids and having varied elastic properties in the through-thickness direction.

The general theory to describe the reflection and transmission phenomena at interfaces of anisotropic elastic media can be found in the reference books of Fedorov [17], Musgrave [18] and Auld [19] for instance. Rokhlin et al. [20] and Lanceleur et al. [21] provided solutions of the reflection and transmission problem for anisotropic elastic plates. Deschamps and Hosten [22] have investigates this problem for orthotropic viscoelastic plates. However, the studies on ultrasound responses are usually limited to media whose the material properties are perfectly known. In this situation, the models used to describe the evaluation techniques are determinism. In the following, the deterministic model is so-called mean model in reference to the mean values given at the material properties. However, most of the time, only partial information is available on these material properties whose the values which are obtained via experimental measurements. Hence, the effective macroscopic properties of the biological tissues depend on many factors (genetic, environmental, physiological and pathological), at various length scales. As consequence, it is useful to consider these parameters as uncertain [23,24].

This study follows the probabilistic framework to analyze wave reflection and transmission through anisotropic elastic plates proposed in [25]. A parametric probabilistic method, which is based on the maximum entropy principle, is used to generate an optimal probabilistic model. The semi-analytical finite element (SAFE) method [26,27] is used to compute the reflection and transmission coefficients of anisotropic heterogeneous solid plates. In the deterministic model from which the stochastic model is constructed, the cortical bone plate is assumed to be homogeneous and transversely isotropic elastic. Whereas in the stochastic model, the plate is anisotropic and heterogeneous with material properties that vary along the thickness direction. Note that the material properties of the fluids and the bone mass density are supposed to be deterministic while the elasticity tensor of bone tissue is randomly varied in the thickness direction. A parametric study is presented, highlighting effect of the uncertainty of elasticity properties on the reflection and transmission coefficients by varying the frequency, angle of incidence and bone thickness.

After this introduction on ultrasound wave reflection and transmission through cortical bone, the remainder of paper is organized as follows. In section 2, the elastoacoustic problem of an anisotropic and heterogeneous elastic plate sandwiched between 
two fluids is formulated. In section 3, a numerical solving method of the problem described by the deterministic model is presented in order to determine the reflection and transmission coefficients. The components of the elasticity tensor are obtained via a probabilistic model which is described in section 4 . In section 5, results are presented for the configuration proposed and a discussion is led about these results. Finally, a conclusion is drawn in section 6 .

\section{PROBLEM FORMULATION}

\subsection{Model specification}

Let us consider a Cartesian reference system $\mathcal{R}\left(O ; \mathbf{e}_{1}, \mathbf{e}_{2}, \mathbf{e}_{3}\right)$, where $\mathrm{O}$ is the origin of the space and $\left(\mathbf{e}_{1}, \mathbf{e}_{2}, \mathbf{e}_{3}\right)$ is the orthonormal basis. The studied problem is an elastic solid layer with constant thickness $h$ that occupies the unbounded domain $\Omega^{b}$ in $\mathbf{e}_{1}$-axis and sandwiched between two fluid half-spaces $\Omega_{1}^{f}$ and $\Omega_{2}^{f}$. The system is excited by oblique plane and harmonic wave $p_{I}$ propagating with an angular frequency $\omega$ at an angle $\gamma$ from fluid domain $\Omega_{1}^{f}$ to the solid layer $\Omega^{b}$ as illustrated in Fig. 1. The interfaces between the elastic layer $\Omega^{b}$ and the fluid media $\Omega_{1}^{f}$ and $\Omega_{2}^{f}$ are assumed to be flat and denoted by $\Gamma_{1}^{b f}$ and $\Gamma_{2}^{b f}$, respectively. Due to the nature of the source and to the geometrical configuration, the elastoacoustic wave motion will be independent of $x_{3}$. Consequently, the coordinate $x_{3}$ is implicit in the mathematical expressions to follow. As result, if the coordinates of a point $M$ are specified by $\left(x_{1}, x_{2}, x_{3}\right)$ in $\mathcal{R}$, the domains $\Omega_{1}^{f}, \Omega_{2}^{f}$ and $\Omega^{b}$ may be defined by

$$
\begin{aligned}
& \Omega_{1}^{f}=\left\{M\left(x_{1}, x_{2}\right) ; x_{2} \geq 0\right\}, \\
& \Omega_{2}^{f}=\left\{M\left(x_{1}, x_{2}\right) ; x_{2} \leq-h\right\}, \\
& \Omega^{b}=\left\{M\left(x_{1}, x_{2}\right) ;-h \leq x_{2} \leq 0\right\} .
\end{aligned}
$$

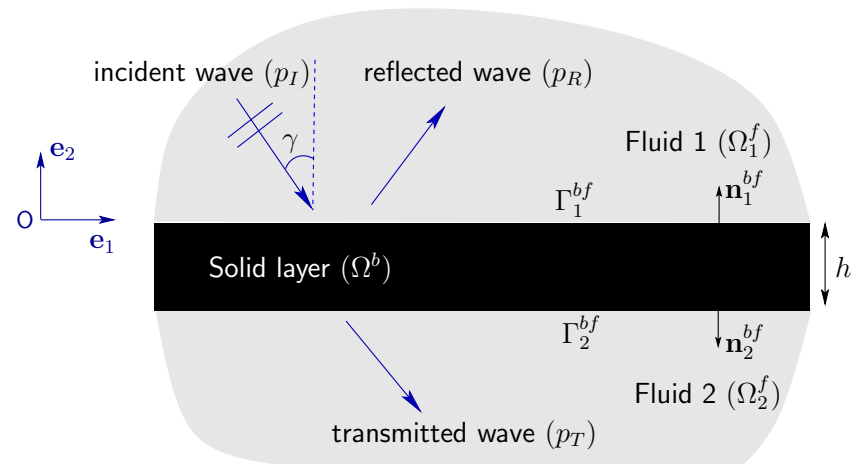

Fig. 1. Schematic representation of an elastic plate surrounded by to fluids and excited by an oblique plane wave in the $\left(O ; \mathbf{e}_{1}, \mathbf{e}_{2}\right)$ plane 
In what follows, we note respectively $\nabla, \nabla \cdot$ and $\nabla^{2}$ the gradient, divergence and Laplacian operators in two-dimensional space (2D). The time derivative is denoted by a dot superimposed. The operators $\partial_{i}$ and $\partial_{i}^{2}$ denote respectively the first and second order partial derivatives with respect to $x_{i}$. The boldface symbols are used to designate the matrices, the fields of vectors and tensors in two-dimensional and three-dimensional spaces.

\subsection{Acoustic field equations of the fluids}

In the context of linearized elasticity theory, and neglecting the body forces, the wave equation in the domain $\Omega_{\alpha}^{f}(\alpha=1,2)$ reads

$$
\frac{1}{c_{\alpha}^{2}} \ddot{p}_{\alpha}-\nabla^{2} p_{\alpha}=0, \quad \forall M \in \Omega_{\alpha}^{f} \quad(\alpha=1,2),
$$

where the celerity and pressure in the fluid are denoted by $c_{\alpha}$ and $p_{\alpha}$, respectively. The velocity vector $\mathbf{v}_{\alpha}(M, t)$ is related to the pressure field $p_{\alpha}(M, t)$ by the Euler equation

$$
\rho_{\alpha} \dot{\mathbf{v}}_{\alpha}+\nabla p_{\alpha}=\mathbf{0}, \quad \forall M \in \Omega_{\alpha}^{f} \quad(\alpha=1,2),
$$

where $\dot{\mathbf{v}}_{\alpha}=\ddot{\mathbf{u}}^{(\alpha)}$ and $\mathbf{u}^{(\alpha)}$ denotes the displacement vector in the fluid. The mass density is denoted by $\rho_{\alpha}$.

\subsection{Governing equations of the elastic solid layer}

Neglecting the body forces, the dynamic equation of motion in the elastic solid layer $\Omega^{b}$ s given by

$$
\rho \ddot{\mathbf{u}}-\nabla \cdot \sigma=\mathbf{0}, \quad \forall M \in \Omega^{b},
$$

which may be written in the vectorial form

$$
\rho \ddot{\mathbf{u}}-\mathbf{L}^{T} \mathbf{s}=\mathbf{0}, \quad \forall M \in \Omega^{b},
$$

in which $\rho$ is denote the mass density of the solid, $\mathbf{u}=\left(u_{1}, u_{2}\right)^{T}$ is the displacement vector in which the superscript " $\mathrm{T}$ " denotes the transpose operator, the vector s contains the components of the stress tensor $\sigma$ and is denoted by $\mathbf{s}=\left(\sigma_{11}, \sigma_{22}, \sigma_{12}\right)^{T}$. The operator $\mathbf{L}$ is defined by

$$
\mathbf{L}=\mathbf{L}_{1} \partial_{1}+\mathbf{L}_{2} \partial_{2}, \quad \mathbf{L}_{1}=\left[\begin{array}{ll}
1 & 0 \\
0 & 0 \\
0 & 1
\end{array}\right], \quad \mathbf{L}_{2}=\left[\begin{array}{ll}
0 & 0 \\
0 & 1 \\
1 & 0
\end{array}\right]
$$

By using the notation of Voigt, the Hooke law reads: $\mathbf{s}=\mathbf{c e}$ where $\mathbf{e}$ is a vector containing the strain components which are given by $\mathbf{e}=\left(\epsilon_{11}, \epsilon_{22}, 2 \epsilon_{12}\right)^{T}=\mathbf{L u}$, and $\mathbf{c}$ is the matrix containing the components of the anisotropic elasticity tensor with the Voigt notation

$$
\mathbf{c}=\left[\begin{array}{lll}
c_{11} & c_{12} & c_{16} \\
c_{12} & c_{22} & c_{26} \\
c_{16} & c_{26} & c_{66}
\end{array}\right]
$$


It is assumed that solid layer $\Omega^{b}$ is homogenous according to the $\mathbf{e}_{1}$-axis, but heterogeneous in the $\mathbf{e}_{2}$-axis. Consequently, the physical properties of the solid only depend on $x_{2}$, i.e. $\mathbf{c}=\mathbf{c}\left(x_{2}\right)$.

\subsection{Boundary conditions}

The continuity conditions of the normal velocity and of the traction at the interfaces require

$$
\begin{aligned}
& \nabla p_{\alpha} \cdot \mathbf{n}_{\alpha}^{b f}=-\rho_{\alpha} \ddot{\mathbf{u}}^{(\alpha)} \cdot \mathbf{n}_{\alpha}^{b f}, \quad \forall M \in \Gamma_{\alpha}^{b f} \quad(\alpha=1,2), \\
& \sigma \mathbf{n}_{\alpha}^{b f}=-p_{\alpha} \mathbf{n}_{\alpha}^{b f},
\end{aligned}
$$

where $\mathbf{n}_{\alpha}^{b f}$ is the outer unit normal vector of $\Omega^{b}$ at the interfaces $\Gamma_{\alpha}^{b f}$. Seeing that $\mathbf{n}_{1}^{b f}=$ $-\mathbf{n}_{2}^{b f}=(0,1)^{T}$, Eqs. (8) may be written as follows

$$
\begin{aligned}
& \partial_{2} p_{\alpha}=-\rho_{\alpha} \ddot{u}_{2}^{(\alpha)}, \quad \forall M \in \Gamma_{\alpha}^{b f} \quad(\alpha=1,2), \\
& \mathbf{t}=\left(0,-p_{\alpha}\right)^{T},
\end{aligned}
$$

in which $\mathbf{t}=\left(\sigma_{12}, \sigma_{22}\right)^{T}=\mathbf{L}_{2}^{T} \mathbf{s}$.

\section{DETERMINISTIC MODEL SOLUTION}

\subsection{Incident, reflected and transmitted waves in the fluids}

Due to the homogeneity of the system along $\mathbf{e}_{1}$-axis, we look for the plane wave solution in the fluids (see Eq. (2)) in the form

$$
p_{\alpha}(M, t)=\hat{p}_{\alpha}\left(x_{2}\right) \exp \left(\mathrm{i}\left(k_{1} x_{1}-\omega t\right)\right), \quad \forall M \in \Omega_{\alpha}^{f}(\alpha=1,2) .
$$

By substituting Eq. (10) into Eqs. (2) and (9) leads to the following equation

$$
\partial_{2}^{2} \hat{p}_{\alpha}+\left(\frac{\omega^{2}}{c_{\alpha}^{2}}-k_{1}^{2}\right) \hat{p}_{\alpha}=0, \quad \forall M \in \Omega_{\alpha}^{f} \quad(\alpha=1,2) .
$$

The incident wave $p_{I}$ gives a reflected wave $p_{R}$ in the midium $\Omega_{1}^{f}$ and a transmitted wave $p_{T}$ in the fluid $\Omega_{2}^{f}$. The solutions in $\Omega_{1}^{f}$ is a superposition of the incident and reflected wave fields of which the amplitudes are denoted by $P_{I}$ and $P_{R}$, respectively

$$
\hat{p}_{1}=P_{I} \exp \left(\mathrm{i} k_{2}^{(1)} x_{2}\right)+P_{R} \exp \left(-\mathrm{i} k_{2}^{(1)} x_{2}\right),
$$

where $i^{2}=-1, k_{1}^{2}+\left(k_{2}^{(1)}\right)^{2}=\left(\omega / c_{1}\right)^{2}=k_{0}^{2}$. The wavenumbers with respect to $\mathbf{e}_{1}$ and $\mathbf{e}_{2}$ are respectively given by: $k_{1}=k_{0} \sin \gamma$ and $k_{2}^{(1)}=-k_{0} \cos \gamma$.

Likewise, the solution of transmitted wave field is given by

$$
\hat{p}_{2}=P_{T} \exp \left(\mathrm{i} k_{2}^{(2)}\left(x_{2}+h\right)\right), \quad \text { where } k_{2}^{(2)}=-\sqrt{\left(\omega / c_{2}\right)^{2}-k_{1}^{2}} .
$$




\subsection{Displacement vector in the solid layer}

The plane wave solution in the solid (see Eq. (5)) is giving in the following form

$$
\mathbf{u}(M, t)=\hat{\mathbf{u}}\left(x_{2}\right) \exp \left[\mathrm{i}\left(k_{1} x_{1}-\omega t\right)\right], \quad \forall M \in \Omega^{b},
$$

where $\hat{\mathbf{u}}=\left(\hat{u}_{1}, \hat{u}_{2}\right)^{T}$. The equation of motion (5) can be written as a system of partial differential equations on the displacement $\hat{\mathbf{u}}$ with respect only to $x_{2}$

$$
\left(-\omega^{2} \mathbf{A}_{1}+k_{1}^{2} \mathbf{A}_{2}\right) \hat{\mathbf{u}}-\mathrm{i} k_{1} \mathbf{A}_{3}^{T} \partial_{2} \hat{\mathbf{u}}-\partial_{2} \mathbf{t}=\mathbf{0}, \quad \forall x_{2} \in[-h, 0],
$$

in which $\mathbf{t}=\left(i k_{1} \mathbf{A}_{3}+\mathbf{A}_{4} \partial_{2}\right) \hat{\mathbf{u}}$, and the two-by-two matrices $\mathbf{A}_{i}(i=1, \ldots, 4)$ are defined by

$$
\mathbf{A}_{1}=\rho \mathbf{I}_{d}, \quad \mathbf{A}_{2}=\mathbf{L}_{1}^{T} \mathbf{c} \mathbf{L}_{1}, \quad \mathbf{A}_{3}=\mathbf{L}_{2}^{T} \mathbf{c L}_{1}, \quad \mathbf{A}_{4}=\mathbf{L}_{2}^{T} \mathbf{c L}_{2},
$$

where $\mathbf{I}_{d}$ is the two-by-two identity matrix. By using the general solutions given by Eqs. (12) and (13), the boundary conditions (9) may be expressed as

$$
\begin{aligned}
& \hat{u}_{2}(0)=\frac{\left(P_{I}-P_{R}\right)}{\beta_{1}}, \quad \hat{u}_{2}(-h)=\frac{P_{T}}{\beta_{2}}, \\
& \mathbf{t}(0)=-\left(0, P_{I}+P_{R}\right)^{T}, \quad \mathbf{t}(-h)=-\left(0, P_{T}\right)^{T},
\end{aligned}
$$

where the constants $\beta_{1}$ and $\beta_{2}$ are given by

$$
\beta_{1}=\frac{\mathrm{i} k_{2}^{(1)}}{\rho_{1} \omega^{2}}, \quad \beta_{2}=\frac{\mathrm{i} k_{2}^{(2)}}{\rho_{2} \omega^{2}} .
$$

By eliminating $P_{R}$ and $P_{T}$ in Eqs. (17) and (18), one may deduce the impedance conditions as follows

$$
\mathbf{t}(0)=\mathbf{F}_{0}-\mathbf{P}_{1} \hat{\mathbf{u}}(0), \quad \mathbf{t}(-h)=\mathbf{P}_{2} \hat{\mathbf{u}}(-h),
$$

where

$$
\mathbf{F}_{0}=\left(\begin{array}{c}
0 \\
-2 P_{I}
\end{array}\right), \quad \mathbf{P}_{1}=\left[\begin{array}{cc}
0 & 0 \\
0 & -\beta_{1}
\end{array}\right], \quad \mathbf{P}_{2}=\left[\begin{array}{cc}
0 & 0 \\
0 & -\beta_{2}
\end{array}\right]
$$

The weak formulation of the problem defined from Eq. (15) may be derived using standard procedure [28]. Let $\mathcal{C}^{\text {ad }}$ be the admissible function space constituted by all sufficient smooth complex-valued functions $\left.x_{2} \in\right]-h, 0\left[\rightarrow \delta \mathbf{u}\left(x_{2}\right) \in \mathbb{C} \times \mathbb{C}\right.$, where $\mathbb{C}$ denotes the set of complex numbers. The conjugate transpose of $\delta \mathbf{u}$ is denoted $\delta \mathbf{u}^{*}$. By multiplying the equation (15) with the test function $\delta \mathbf{u}^{*} \in C^{a d}$ and integrating by parts, we obtain

$$
\int_{-h}^{0} \delta \mathbf{u}^{*}\left(-\omega^{2} \mathbf{A}_{1}+k_{1}^{2} \mathbf{A}_{2}-\mathrm{i} k_{1} \mathbf{A}_{3}^{T} \partial_{2}\right) \hat{\mathbf{u}} d x_{2}+\int_{-h}^{0} \partial_{2}\left(\delta \mathbf{u}^{*}\right) \mathbf{t} d x_{2}-\left[\delta \mathbf{u}^{*} \mathbf{t}\right]_{-h}^{0}=0
$$

The last term in Eq. (22) may be calculated by using the boundary conditions (20). Thus the weak formulation of the problem is: Find $\mathbf{u}\left(x_{2}\right) \in \mathcal{C}^{\text {ad }}$ such that

$$
\begin{aligned}
\int_{-h}^{0} \delta \mathbf{u}^{*}\left(-\omega^{2} \mathbf{A}_{1}+k_{1}^{2} \mathbf{A}_{2}-\mathrm{i} k_{1} \mathbf{A}_{3}^{T} \partial_{2}\right) \hat{\mathbf{u}} d x_{2}+\int_{-h}^{0} \partial_{2}\left(\delta \mathbf{u}^{*}\right)\left(\mathrm{i} k_{1} \mathbf{A}_{3}+\mathbf{A}_{4} \partial_{2}\right) \hat{\mathbf{u}} d x_{2} \\
+\delta \mathbf{u}^{*}(0) \mathbf{P}_{1} \hat{\mathbf{u}}(0)+\delta \mathbf{u}^{*}(-h) \mathbf{P}_{2} \hat{\mathbf{u}}(-h)=\delta \mathbf{u}^{*} \mathbf{F}_{0}
\end{aligned}
$$


for all $\delta \mathbf{u}^{*} \in C^{a d}$. In order to solve the problem (see Eq. (23)) by using the FEM, we discretize the domain $[-h, 0]$ into a mesh which contains $n^{e l}$ elements $\Omega_{e}:[-h, 0]=\bigcup_{e}(e=$ $\left.1, \ldots, n^{e l}\right)$. Using the Galerkin method, the two functions $\mathbf{u}$ and $\delta \mathbf{u}$ in each element $e$ are approximated using the same interpolation function

$$
\hat{\mathbf{u}}\left(x_{2}\right)=\mathbf{N}_{e} \mathbf{U}_{e}, \quad \delta \mathbf{u}\left(x_{2}\right)=\mathbf{N}_{e} \delta \mathbf{U}_{e}, \quad \forall x_{2} \in \Omega_{e},
$$

where $\mathbf{N}_{e}$ is the interpolation function, $\mathbf{U}_{e}$ and $\delta \mathbf{U}_{e}$ are respectively the vectors of nodal solutions of $\mathbf{u}$ and $\delta \mathbf{u}$ in $\Omega_{e}$. Substituting Eq. (24) into Eq. (23) and assembling the elementary matrices, we obtain the following system of linear equations

$$
\left(\mathbf{K}+\mathbf{K}^{\Gamma}\right) \mathbf{U}=\mathbf{F},
$$

where $\mathbf{U}$ is the global nodal displacement vector, $\mathbf{K}$ is the global "stiffness matrix" of the solid, $\mathbf{K}^{\Gamma}$ represents the coupling operator between the fluid and the solid, and $\mathbf{F}$ is the force vector

$$
\begin{aligned}
& \mathbf{K}=-\omega^{2} \mathbf{K}_{1}+k_{1}^{2} \mathbf{K}_{2}+\mathrm{i} k_{1} \mathbf{K}_{3}+\mathbf{K}_{4}, \\
& \mathbf{K}^{\Gamma}=\operatorname{Diag}\left\{0,-\frac{\rho_{2} \omega^{2}}{\beta_{2}}, 0, \ldots, 0,-\frac{\rho_{1} \omega^{2}}{\beta_{1}}\right\}, \\
& \mathbf{F}=\left\{0, \ldots, 0,-2 P_{I}\right\}^{T},
\end{aligned}
$$

and

$$
\begin{aligned}
& \mathbf{K}_{1}=\bigcup_{e} \int_{\Omega_{e}} \mathbf{N}_{e}^{T} \mathbf{A}_{1} \mathbf{N}_{e} d x_{2}, \quad \mathbf{K}_{2}=\bigcup_{e} \int_{\Omega_{e}} \mathbf{N}_{e}^{T} \mathbf{A}_{2} \mathbf{N}_{e} d x_{2}, \\
& \mathbf{K}_{3}=\bigcup_{e} \int_{\Omega_{e}} 2\left[\mathbf{N}_{e}^{\prime T} \mathbf{A}_{3} \mathbf{N}_{e}\right]_{a} d x_{2}, \quad \mathbf{K}_{4}=\bigcup_{e} \int_{\Omega_{e}} \mathbf{N}_{e}^{\prime T} \mathbf{A}_{4} \mathbf{N}_{e}^{\prime} d x_{2},
\end{aligned}
$$

where the notation $[\star]_{a}$ denotes the antisymmetric part of $[\star]$ and $\star^{\prime}$ the derivative with respect to $x_{2}$. In this study, Gauss quadrature rule has been used for computing the integrations over the elements.

\subsection{Reflection and transmission coefficients}

Generally, the reflection and transmission coefficients are defined as the ratio between the amplitudes of the reflected and transmitted waves to the amplitude of the incident wave, respectively. These coefficients may be computed by using (see Eqs. (17) and (18))

$$
R=\left|\frac{P_{R}}{P_{I}}\right|=\left|1-\frac{\beta_{1} \hat{u}_{2}(0)}{P_{I}}\right|, \quad T=\left|\frac{P_{T}}{P_{I}}\right|=\left|\frac{\beta_{2} \hat{u}_{2}(-h)}{P_{I}}\right|,
$$

in which $\hat{u}_{2}(0)$ and $\hat{u}_{2}(-h)$ are obtained by solving Eq. (25).

\section{STOCHASTIC MODEL SOLUTION}

\subsection{Probabilistic model of uncertainties for the elasticity tensor}

This section provides a brief description of the probabilistic model of random elastic matrix. We only sketch out the main features of the model in the context of this study. This model is proposed by Soize [29] for the construction of the random elastic tensor in 
order to describe the random heterogeneity in the solid layer along the $\mathbf{e}_{2}$ direction. The maximum entropy principle $[30,31]$ and the random matrix theory [32] are used in three dimension to describe the model. Although, the random elasticity tensor $(n \times n)$ has been generated in three dimensions $(n=6)$ with a high value of the spatial correlation lengths in the $\mathbf{e}_{1}$ and $\mathbf{e}_{3}$ directions, only heterogeneous six components, which correspond to the ones defined in the plane $\left(\mathbf{e}_{1}, \mathbf{e}_{2}\right)$, are extracted to be used for the simulations. Indeed, the solid layer is assumed to be invariant in the $\mathbf{e}_{3}$-direction and homogeneous in the $\mathbf{e}_{1}$-direction.

We denote by $\mathbf{C}\left(x_{2}\right) \in \mathbf{M}_{n}^{+}(\mathbb{R})$ the random elastic matrix at $x_{2}$ and by $\mathbf{c}\left(x_{2}\right) \in$ $\mathbf{M}_{n}^{+}(\mathbb{R})$ its mean value, respectively, and for which the following relationship is satisfied $\mathbb{E}\left\{\mathbf{C}\left(x_{2}\right)\right\}=\mathbf{c}\left(x_{2}\right)$, where $\mathbb{E}\{\star\}$ designates the mathematical expectation, $\mathbf{M}_{n}^{+}(\mathbb{R})$ the set of all the $(n \times n)$ real symmetric positive-definite matrices and $\mathbb{R}$ the set of real numbers. The matrix $\mathbf{c}\left(x_{2}\right)$ can be decomposed into a product of a unique upper triangular real matrix $\mathcal{L}\left(x_{2}\right)$ with strictly positive diagonal entries and its transpose

$$
\mathbf{c}\left(x_{2}\right)=\mathcal{L}^{T}\left(x_{2}\right) \mathcal{L}\left(x_{2}\right) \text {. }
$$

The random matrix $\mathbf{C}\left(x_{2}\right)$ is parameterized by its mean value $\mathbf{c}\left(x_{2}\right)$, the dispersion level $\delta$ and the correlation length $\lambda$ in the $\mathbf{e}_{2}$-direction, which is denoted by $\mathbf{C}\left(x_{2} ; \mathbf{c}, \delta, \lambda\right)$ and may be decomposed into the following form

$$
\mathbf{C}\left(x_{2} ; \mathbf{c}, \delta, \lambda\right)=\mathcal{L}^{T}\left(x_{2}\right) \mathbf{G}\left(x_{2} ; \mathbf{c}, \delta, \lambda\right) \mathcal{L}\left(x_{2}\right),
$$

where $\mathbf{G}\left(x_{2} ; \mathbf{c}, \delta, \lambda\right)$, called the stochastic germ matrix, is a homogeneous and normalized non-Gaussian positive-definite matrix-valued second-order random field with values in $\mathbb{M}_{n}^{+}(\mathbf{R})$. The dispersion parameter $\delta$, which is a scalar, controls the dispersion of the random matrix $\mathbf{G}\left(x_{2} ; \mathbf{c}, \delta, \lambda\right)$ and must satisfy the following inequality $0<\delta<$ $\sqrt{(n+1) /(n+5)}$, which allows the mean-square convergence condition for the germ matrix to be hold [33]. It is proved that the dispersion parameter $\delta$ is related to a parameter $\delta_{C}$ which evaluates the dispersion of the random matrix $\mathbf{C}\left(x_{2}\right)$ by the relation given by

$$
\delta_{C}\left(x_{2}\right)=\frac{\delta}{\sqrt{n+1}}\left\{1+\frac{\left\{\operatorname{Tra}\left(\mathbf{c}\left(x_{2}\right)\right)\right\}^{2}}{\operatorname{Tra}\left(\left[\mathbf{c}\left(x_{2}\right)\right]^{2}\right)}\right\}^{1 / 2} .
$$

The correlation length $\lambda$, which is a scalar, is a measure of the distance up to which one has spatial memory of the spatial variations in the material properties.

\subsection{Stochastic solver}

\subsubsection{Reflection and transmission coefficients}

The Monte Carlo simulation is used for the reliability analysis. For a given parameter set $\mathbf{c}, h, \delta$ and $\lambda$, we begin with the discretization of the $1 \mathrm{D}$ domain $[-h, 0]$ by using quadratic Lagrangian elements. The global $x_{2}$-coordinates of the Gauss points in all elements are denoted by $x_{2}^{k}$ for $k=1, \ldots, n_{g p}$, where $n_{g p}$ is the total number of the Gauss points in the mesh. 
Let $n_{r}$ be the total number of realizations, we may construct the set of the independent realizations $\phi_{j}$, for $j=1, \ldots, n_{r}$, defined by

$$
\mathcal{S}=\left\{\left\{\mathbf{C}\left(x_{2}^{k}, \phi_{1}\right)\right\}_{k=1, \ldots, n_{p g}}, \ldots,\left\{\mathbf{C}\left(x_{2}^{k}, \phi_{n_{r}}\right)\right\}_{k=1, \ldots, n_{p g}}\right\}
$$

in which $\left\{\mathbf{C}\left(x_{2}^{k}, \phi_{j}\right)\right\}_{k=1, \ldots, n_{p g}}$ are $n_{p g}$ samples at points $x_{2}^{k}$ of one realization of random matrices field $\mathbf{C}\left(x_{2} ; \mathbf{c}, \delta, \lambda\right)$ as described in Section 4.1 (see [29] and [34]).

For each statistical independent realization $\phi_{j}, n_{r}$ statistical independent realizations of the random reflection and transmission coefficients, $R\left(\phi_{j}\right)$ and $T\left(\phi_{j}\right)$ respectively, may be calculated following the SAFE procedure presented in Section 3.

\subsubsection{Convergence analysis}

Convergence analysis with respect to the number of realizations $n_{r}$ may be performed by studying the convergence of statistical estimates of the second-order moments of $R$ and $T$, respectively denoted by $\mathrm{m}_{R, 2}=\mathbb{E}\left\{R^{2}\right\}$ and $\mathrm{m}_{T, 2}=\mathbb{E}\left\{T^{2}\right\}$. The statistical estimates $\operatorname{Conv}_{R}\left(n_{r}\right)$ and $\operatorname{Conv}_{T}\left(n_{r}\right)$ of the second-order moments of $R$ and $T$ respectively are defined by

$$
\operatorname{Conv}_{R}\left(n_{r}\right)=\left(\frac{1}{n_{r}} \sum_{j=1}^{n_{r}} R^{2}\left(\phi_{j}\right)\right)^{1 / 2}, \operatorname{Conv}_{T}\left(n_{r}\right)=\left(\frac{1}{n_{r}} \sum_{j=1}^{n_{r}} T^{2}\left(\phi_{j}\right)\right)^{1 / 2} .
$$

\subsubsection{Confidence regions}

The confidence regions of both random variables $R$ and $T$ is carried out in using a procedure based on the quantile method. The confidence region of $T$ is limited by a lower envelope and an upper envelope, denoted by $t^{-}$and $t^{+}$, respectively

$$
\mathbb{P}\left(t^{-}<T \leq t^{+}\right)=P_{c}
$$

where $\mathbb{P}(A)$ denotes the probability measure of an event $A$.

Let $F_{T}$ be the cumulative distribution function of $T$ defined by $F_{T}(t)=\mathbb{P}(T \leq t)$. The $p$-th quantile $(p \in] 0,1[)$ of $F_{T}$ is defined by

$$
\zeta(p)=\inf \left\{t: F_{T}(t) \geq p\right\} .
$$

The lower and upper envelopes $t^{-}$and $t^{+}$are defined by

$$
t^{-}=\zeta\left(\frac{1-P_{c}}{2}\right), \quad t^{+}=\zeta\left(\frac{1+P_{c}}{2}\right) .
$$

Let $\tilde{t}_{1}<\ldots<\tilde{t}_{n}$ be the order statistics associated with $T\left(\phi_{1}\right), \ldots, T\left(\phi_{n_{r}}\right)$, we have the following estimations

$$
\begin{aligned}
& t^{-} \simeq \tilde{t}_{j^{-}}, \quad j^{-}=\operatorname{fix}\left[n_{r}\left(1-P_{c}\right) / 2\right], \\
& t^{+} \simeq \tilde{t}_{j^{+}}, \quad j^{+}=\operatorname{fix}\left[n_{r}\left(1+P_{c}\right) / 2\right],
\end{aligned}
$$

in which fix $(z)$ denotes the integer part of the real number $z$. 


\section{RESULTS AND DISCUSSION}

\subsection{Validation}

\subsubsection{Numerical parameters}

Parameters for the mean model. In this model, the solid and the fluid layers represent bone and soft tissues (soft tissue and marrow), respectively. The bone phantom has a constant thickness $h$ and is submerged in water. The mechanical properties of the fluid $\left(\rho_{1}=\rho_{2}\right.$ and $\left.c_{1}=c_{2}\right)$ and the bone are shown in Tab. 1 , which are taken from the mechanical test results obtained by [35]. Note that the other components of the elasticity matrix are given by: $c_{22}=c_{33}, c_{12}=c_{13}=c_{21}=c_{31}, c_{23}=c_{32}$ and $c_{55}=c_{66}$.

Table 1. Mechanical parameters for the mean model

\begin{tabular}{|c|c|c|c|c|c|c|c|}
\hline \multicolumn{2}{|c|}{ Fluid material properties } & \multicolumn{6}{|c|}{ Solid material properties } \\
\hline $\begin{array}{c}\rho_{1}=\rho_{2} \\
\left(\mathrm{~kg} . \mathrm{m}^{-3}\right)\end{array}$ & $\begin{array}{c}c_{1}=c_{2} \\
\left(\mathrm{~m} . \mathrm{s}^{-1}\right)\end{array}$ & $\begin{array}{c}\rho \\
\left(\mathrm{kg} \cdot \mathrm{m}^{-3}\right)\end{array}$ & $\begin{array}{c}c_{11} \\
(\mathrm{GPa})\end{array}$ & $\begin{array}{c}c_{22} \\
(\mathrm{GPa})\end{array}$ & $\begin{array}{c}c_{12} \\
(\mathrm{GPa})\end{array}$ & $\begin{array}{c}c_{66} \\
(\mathrm{GPa})\end{array}$ & $\begin{array}{c}c_{16}=c_{26} \\
(\mathrm{GPa})\end{array}$ \\
\hline 1000 & 1500 & 1722 & 23.05 & 15.10 & 8.71 & 4.7 & 0 \\
\hline
\end{tabular}

Parameters for the uncertain elasticity model. the dispersion $\delta$ and one correlation length $\lambda$ need to be introduced to control the statistical fluctuations of the elasticity field in the $\mathbf{e}_{2}$-direction. A fixed correlation length $\lambda=0.1 \mathrm{~mm}$ will be used, which may be seen as a center-to-center distance between osteons in cortical bone (see [36-38]). Three different values of the dispersion $\delta=0.1,0.2$ and 0.3 will be investigated. The coefficient $\delta_{C}$ which evaluates the dispersion of the random field $\mathbf{C}$ is defined by Eq. (33).

\subsubsection{Stochastic convergence analysis}

The stochastic convergence analysis with respect to number of realizations $n_{r}$ is carried out in studying the convergence of the estimated second-order moment. This convergence lead to determine the minimal number of realizations by the Monte Carlo solver to calculate in order to do the statistics on the interest quantities given by $R$ nd $T$. We consider a $1 \mathrm{~mm}$-thickness solid layer meshed by 200 quadratic elements which allows us to satisfy the requirement that at least 5 elements per correlation length $(\lambda=0.1 \mathrm{~mm})$ are needed. Fig. 2 shows the graphs of functions $n_{r} \rightarrow \operatorname{Conv}_{R}\left(n_{r}\right)$ and $n_{r} \rightarrow \operatorname{Conv}_{T}\left(n_{r}\right)$ in the case of normal incidence $\left(\gamma=0^{\circ}\right)$ for different dispersion $(\delta=0.1, \delta=0.2$ and $\delta=0.3)$. Convergence is reached for $n_{r} \geq 200, n_{r} \geq 400$ and $n_{r} \geq 600$ for the three cases, respectively.

Fig. 3(a) shows an illustration of a realization of the spatial variation of the components of the random field $\mathbf{C}\left(x_{2}\right)$ with respect to $x_{2}$ when $\delta=0.3$. The probability density function may also be seen in Fig. $3 \mathrm{~b}$ in which the random elasticity matrix components $\mathrm{C}\left(x_{2}\right)$ on the interface $\Gamma_{1}^{b f}$ are computed and presented. These functions are obtained on the boundary $\Gamma_{1}^{b f}$ when $\delta=0.3$. One may verify that the mean values of the C's components are equal to the values given for the mean model. In particular, the mean of $c_{16}$ and $c_{26}$ are equal to zero (transversely isotropic model). 


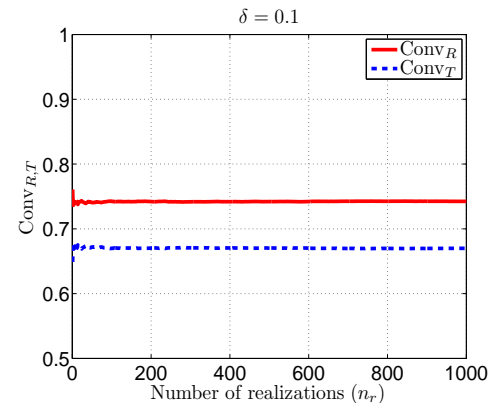

(a)

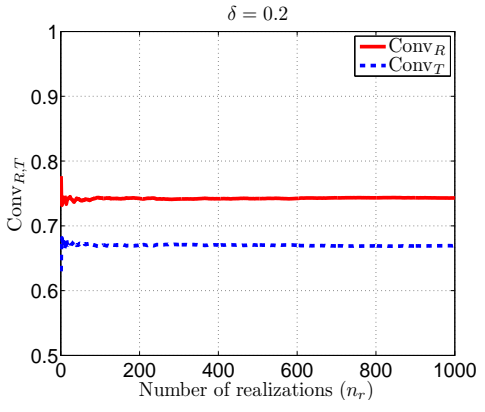

(b)

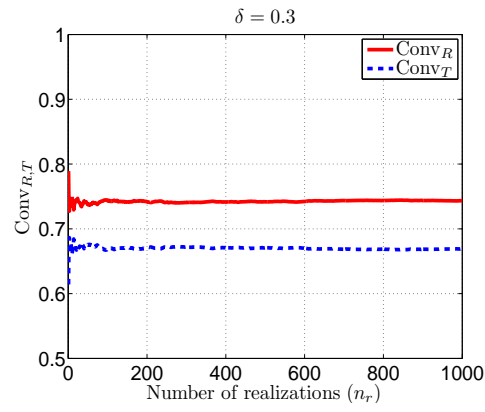

(c)

Fig. 2. (Color online) Stochastic convergence analysis for the case $\gamma=0^{\circ}$ and $f=1 \mathrm{MHz}$. Graphs of functions $n_{r} \rightarrow \operatorname{Conv}_{R}\left(n_{r}\right)$ and $n_{r} \rightarrow \operatorname{Conv}_{T}\left(n_{r}\right)$. The solid and dashed lines are respectively associated with the functions $\operatorname{Conv}_{R}\left(n_{r}\right)$ and $\operatorname{Conv}_{T}\left(n_{r}\right)$. (a) $\delta=0.1$; (b) $\delta=0.2$; (c) $\delta=0.3$

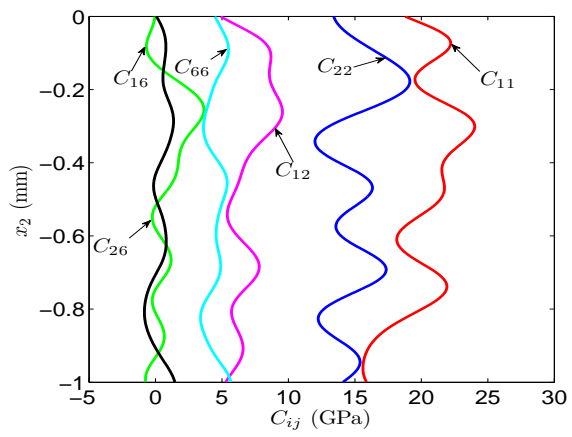

(a)

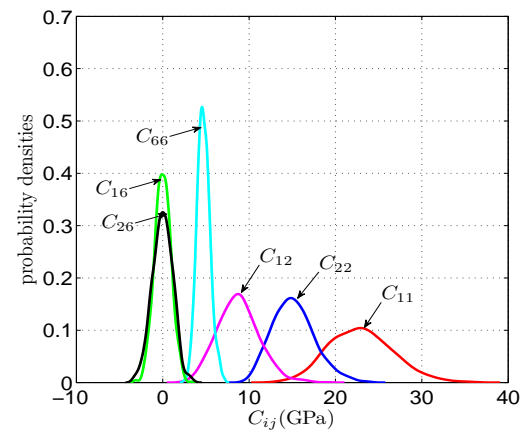

(b)

Fig. 3. (Color online) (a): $x_{2}$-profiles of $C_{i j}$ components when $\delta=0.3$. (b): Probability density functions of the components of the random elastic tensor on boundaries $\Gamma_{1}^{b f}$ when $\delta=0.3$

\subsection{Effects of the level fluctuation of elasticity tensor}

In this section, we investigate the effects of the dispersion parameter $\delta$ which allows to control the statistical fluctuation level of bone elasticity. Fig. 4 illustrates the confidence regions of both reflection and transmission coefficients for a probability level $P_{c}=0.95$. The mean values of these coefficients are represented by a thick line (black line, color online) while the ones obtained with the deterministic model are represented by a dashed line (red line, color online). As illustrated in Fig. 4, the increase of the dispersion parameter has a direct influence on the expanse of the confidence regions. One may also note that the upper envelope and lower envelope of the reflection coefficient are almost symmetric with respect to the mean value while those of transmission coefficient are not symmetrical. 


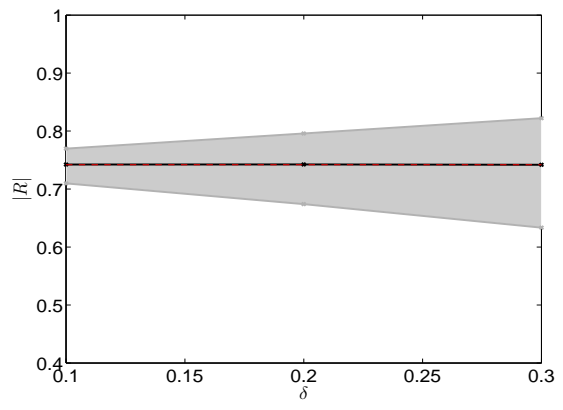

(a)

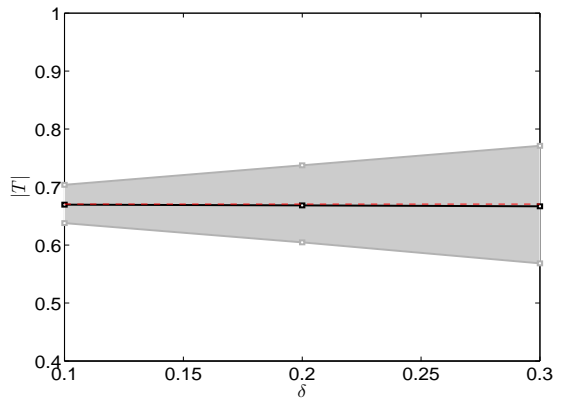

(b)

Fig. 4. (Color online) Confidence region (colored in gray domain) associated with a probability level $P_{c}=0.95$ and mean values (thick and black line) of reflection and transmission coefficients with respect to the dispersion coefficient $\delta$ of random elasticity tensor. The coefficients $R$ and $T$ obtained with the deterministic model are plotted with the thin lines (red line). (a) reflection coefficient $R$; (b) transmission coefficient $T$. These results are obtained with $f=2 \mathrm{MHz}$ and $h=1 \mathrm{~mm}$

\subsection{Effects of incident wave characteristics}

Angle of incidence. The confidence regions associated with a probability level $P_{c}$ for random fields $R$ and $T$ are calculated with respect to the angle of incidence. Figs. 5 and 6 represent confidence regions associated with a probability level $P_{c}=0.95$ of random fields $\gamma \longrightarrow\{R(\gamma)\}$ and $\gamma \rightarrow\{T(\gamma)\}$.

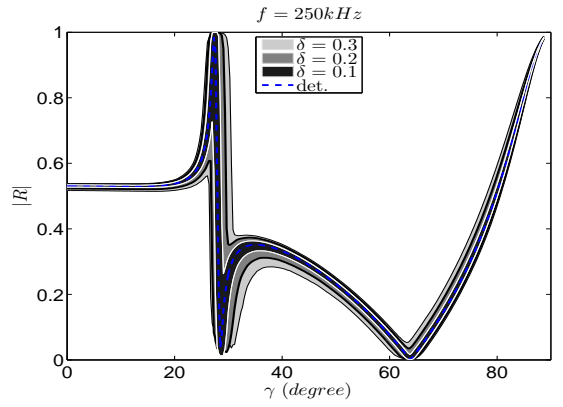

(a)

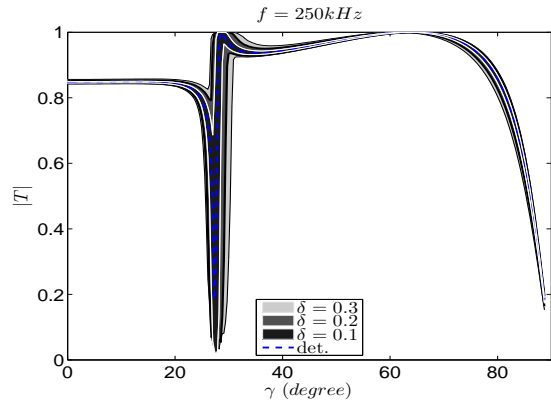

(b)

Fig. 5. Confidence regions of the reflection and transmission coefficients when $f=250 \mathrm{kHz}$. The dotted line represents the deterministic solutions of $R$ and $T$

The upper and lower bounds are constructed using Eqs. (39) and (40). Two similar solid lines represent the functions $\gamma \rightarrow\left\{r^{-}(\gamma)\right\}$ and $\gamma \rightarrow\left\{r^{+}(\gamma)\right\}\left(\gamma \rightarrow\left\{t^{-}(\gamma)\right\}\right.$ and $\gamma \rightarrow\left\{t^{+}(\gamma)\right\}$, respectively) delimiting the confidence region of the reflection coefficient (transmission coefficient, respectively) obtained with the stochastic model when $\delta=0.1$ (black region), $\delta=0.2$ (dark gray region) and $\delta=0.3$ (light gray region). The dotted line represents the function $\gamma \rightarrow\{R(\gamma)\}(\gamma \rightarrow\{T(\gamma)\}$ respectively) obtained with the deterministic model. It is observed that the expanse of the confidence interval is not 


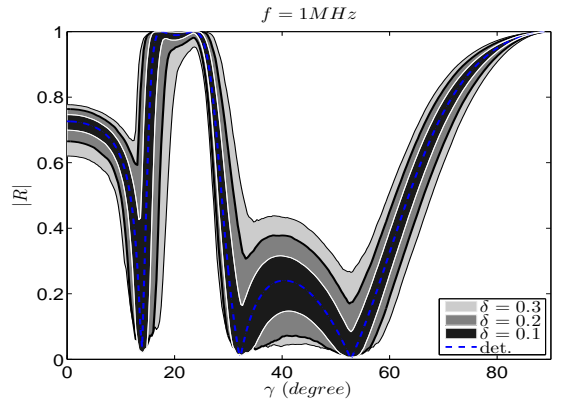

(a)

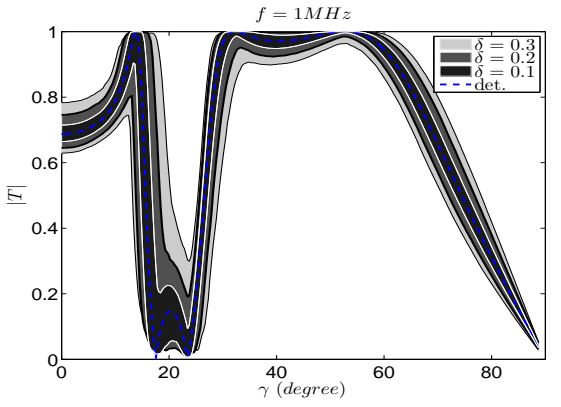

(b)

Fig. 6. Confidence regions of the reflection and transmission coefficients when $f=1 \mathrm{MHz}$. The dotted line represents the deterministic solutions of $R$ and $T$

constant over all incidence angles $\gamma$ increases with the dispersion $\delta$. We may notice that the difference between the mean values of reflection and transmission coefficients (figure is not shown here) and the ones obtained by the mean model is not significant.

In Figs. 7, the probability density functions of the reflection and transmission coefficients are presented for $f=1 \mathrm{MHz}$ when $\delta=0.1$. For each frequency and each coefficient of reflection and transmission, the probability density function is plotted for four incidence angles $\gamma=0^{\circ}, 30^{\circ}, 45^{\circ}$ and $60^{\circ}$. The findings show that the dispersion and the asymmetry degree of the probability density functions increase with $f$ for all incidence angles.

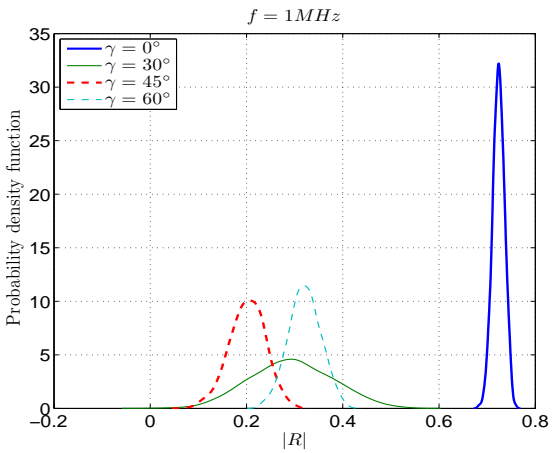

(a)

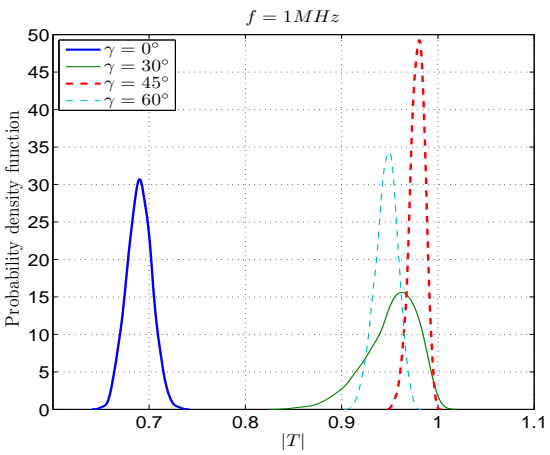

(b)

Fig. 7. (Color online) Probability density function for four incidence angles given by $\theta=0^{\circ}, 30^{\circ}, 45^{\circ}$ and $60^{\circ}$ when $\delta=0.1$. (a) reflection coefficient $R$; (b) transmission coefficient $T$

Frequency. Fig. 8 shows the confidence regions of the reflection and transmission coefficients versus frequency for the incidence angle $\gamma=0^{\circ}$ and $\gamma=30^{\circ}$ when $\delta=0.1$. The gray colored area presents the confidence regions while the reflection and transmission coefficients obtained via the deterministic model are plotted in dashed line. As illustrated in Fig. 8, the expanse of the confidence region increases with frequency. We may also notice that some peaks may be observed on the envelops characterizing the confidence regions of both coefficients of reflection and transmission. Indeed, these peaks are due 


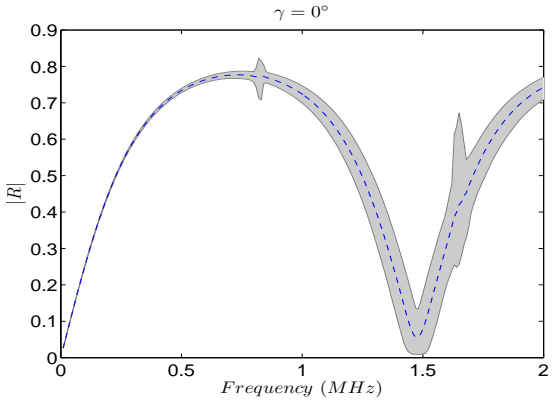

(a)

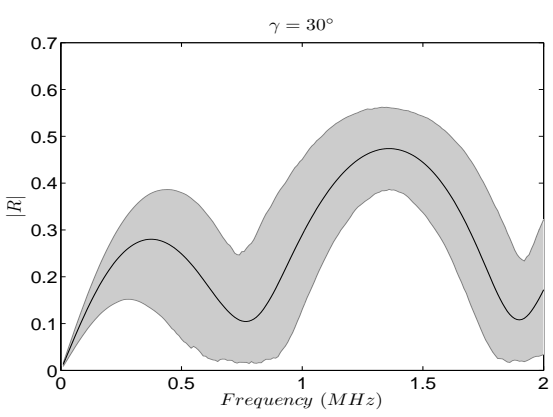

(c)

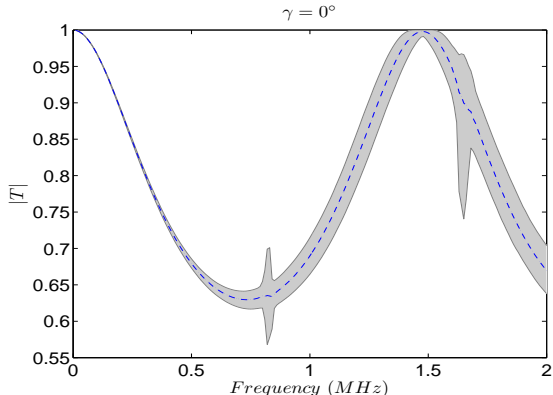

(b)

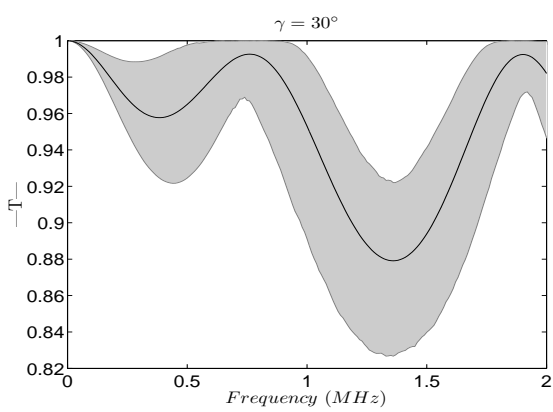

(d)

Fig. 8. Confidence regions of reflection and transmission coefficients with respect to frequency when $\delta=0.1$ and $h=1 \mathrm{~mm}$. The confidence regions are colored in grey while the reflection and transmission coefficients obtained via the deterministic model are plotted in thick solid line. (a) and (c) reflection coefficients $R$ when $\gamma=0^{\circ}$ and $\gamma=30^{\circ}$, respectively; (b) and (d) transmission coefficients $T$ when $\gamma=0^{\circ}$ and $\gamma=30^{\circ}$, respectively

to the shear modes that may exist when the material properties become fully anisotropic. Note that similar remark have been observe in the studying of thick random plate by Nguyen et al [25]. For an inclined angle of incidence $\gamma=30^{\circ}$, the widths of confidence regions of both $R$ and $T$ becomes important, which means that the heterogeneity in the thickness's direction may leads to significant misunderstanding of the acoustic response.

\section{CONCLUSION}

A probabilistic study has been performed to investigate the ultrasonic response of cortical bone plates. It has been shown that both reflection and transmission coefficients are very sensitive to the dispersion parameter which represents the random fluctuation level of elastic properties in the plate, even when the bone's thickness is small $(h=1 \mathrm{~mm})$. It has been shown frequency and the angle of incidence have a significant influence on the quantities of interest. The effects of random properties may become much much more important in higher frequencies. The parametric studies also shown that the confidence regions may be less large or larger depending on the angle of incidence, which can be 
useful to develop a strategy in the experimental method of measurement of the reflection and transmission coefficients in order to minimize the errors due to heterogeneity. All these results have great importance on the identification of material properties by solving an inverse problem which will be presented in forthcoming publications. Furthermore, stochastic modeling of reflection and transmission phenomena at interfaces of another kinds of media such as poroelastic or second gradient materials $[39,40]$ will also be investigated.

\section{REFERENCES}

[1] J. L. Williams. Ultrasonic wave propagation in cancellous and cortical bone: prediction of some experimental results by Biot's theory. The Journal of the Acoustical Society of America, 91, (2), (1992), pp. 1106-1112. doi:10.1121/1.402637.

[2] P. Laugier, F. Padilla, F. Peyrin, K. Raum, A. Saied, M. Talmant, and L. Vico. Apport des ultrasons dans l'exploration du tissu osseux. ITBM-RBM, 26, (5-6), (2005), pp. 299 - 311. doi:10.1016/j.rbmret.2005.07.002.

[3] M. Sasso, G. Haiat, Y. Yamato, S. Naili, and M. Matsukawa. Frequency dependence of ultrasonic attenuation in bovine cortical bone: An in vitro study. Journal of Biomechanics, 41, (41), (2008), pp. 347 - 355. doi:10.1016/j.ultrasmedbio.2007.05.022.

[4] C. M. Langton, A. Ali, C. M. Riggs, J. A. Evans, and W. Bonfield. A contact method for the assessment of ultrasonic velocity and broadband attenuation in cortical and cancellous bone. Clinical Physics and Physiological Measurement, 11, (1990), pp. 243-249. doi:10.1088/01430815/11/3/007.

[5] P. H. F. Nicholson, X. G. Muller, R. Cheng, P. Ruegsegger, G. Van der Perre, J. Dequeker, and S. Boonen. Quantitative ultrasound and trabecular architecture in the human calcaneus. Journal of Bone and Mineral Research, 16, (10), (2001), pp. 1886-1892. doi:10.1359/jbmr.2001.16.10.1886.

[6] G. Lowet and G. Van der Perre. Ultrasound velocity measurements in long bones: measurement method and simulation of ultrasound wave propagation. Journal of Biomechanics, 29, (1996), pp. 1255-1262. doi:10.1016/0021-9290(96)00054-1.

[7] E. Bossy, M. Talmant, and P. Laugier. Effect of bone cortical thickness on velocity measurements using ultrasonic axial transmission: A 2D simulation study. The Journal of the Acoustical Society of America, 112, (2002), pp. 297-307. doi:10.1121/1.1480836.

[8] P. Nicholson, P. Moilanen, T. Karkkainen, J. Timonen, and S. Cheng. Guided ultrasonic waves in long bones: modelling, experiment and in vivo application. Physiological Measurement, 23, (4), (2002), pp. 755-768. doi:10.1088/0967-3334/23/4/313.

[9] C. Baron and S. Naili. Propagation of elastic waves in a fluid-loaded anisotropic functionally graded waveguide: Application to ultrasound characterization. The Journal of the Acoustical Society of America, 127, (3), (2010), pp. 1307-1317. doi:10.1121/1.3292949.

[10] L. Le, Y. Gu, Y. Li, and C. Zhang. Probing long bones with ultrasonic body waves. Applied Physics Letters, 96, (2010), p. 114102. doi:10.1063/1.3300474.

[11] D. Ta, W. Wang, K. Huang, Y. Wang, and L. H. Le. Analysis of frequency dependence of ultrasonic backscatter coefficient in cancellous bone. The Journal of the Acoustical Society of America, 124, (6), (2008), pp. 4083-4090. doi:10.1121/1.3001705.

[12] Z. Zheng, L. Le, M. Sacchi, D. Ta, and E. Lou. Spectral ratio method to estimate broadband ultrasound attenuation of cortical bones in vitro using multiple reflections. Physics in Medicine and Biology, 52, (19), (2007), p. 5855. doi:10.1088/0031-9155/52/19/008. 
[13] V. Bousson, A. Meunier, C. Bergot, E. Vicaut, M. Rocha, M. Morais, A. Laval-Jeantet, and J. Laredo. Distribution of intracortical porosity in human midfemoral cortex by age and gender. Journal of Bone and Mineral Research, 16, (2001), pp. 1308-11317. doi:10.1359/jbmr.2001.16.7.1308.

[14] C. Thomas, S. A. Feik, and J. Clement. Regional variation of intracortical porosity in the midshaft of the human femur: age and sex differences. Journal of Anatomy, 206, (2005), pp. 115125. doi:10.1111/j.1469-7580.2005.00384.x.

[15] N. X. Dong and E. X. Guo. The dependence of transverse isotropic elasticity of human femoral cortical boneon porosity. Journal of Biomechanics, 37, (8), (2004), pp. 1281-1287. doi:10.1016/j.jbiomech.2003.12.011.

[16] C. Baron, M. Talmant, and P. Laugier. Effect of porosity on effective diagonal stiffness coefficients $\left(c_{i i}\right)$ and anisotropy of cortical bone at $1 \mathrm{MHz}$ : A finite difference time domain study. The Journal of the Acoustical Society of America, 122, (2007), pp. 1810-1817. doi:10.1121/1.2759165.

[17] F. Fedorov. Theory of elastic waves in crystals. Plenum Press, (1968).

[18] M. Musgrave. Crystal acoustics. Holden Day, San Francisco, (1970).

[19] B. Auld. Acoustic field and waves in solids, Vol. I and II. Krieger Publishing, Malabar, 2nd edition, (1990).

[20] S. I. Rokhlin, T. Bolland, and L. Adler. Reflection and refraction of elasticw aves on a plane interface between two generally anisotropic media. The Journal of the Acoustical Society of America, 79, (1986), pp. 906-918. doi:10.1121/1.393764.

[21] P. Lanceleur, H. Ribeiro, and J.-F. De Belleval. The use of inhomogeneous waves in the reflection-transmission problem at a plane interface between two anisotropic media. The Journal of the Acoustical Society of America, 93, (1993), pp. 1882-1892. doi:10.1121/1.406703.

[22] M. Deschamps and B. Hosten. The effects of viscoelasticity on the reflection and transmission of ultrasonic waves by an orthotropic plate. The Journal of the Acoustical Society of America, 91, (4), (1992), pp. 2007-2015. doi:10.1121/1.403685.

[23] C. Desceliers, C. Soize, Q. Grimal, G. Haiat, and S. Naili. A time-domain method to solve transient elastic wave propagation in a multilayer medium with a hybrid spectral-finite element space approximation. Wave Motion, 45, (4), (2008), pp. 383 - 399. doi:10.1016/j.wavemoti.2007.09.001.

[24] C. Desceliers, C. Soize, Q. Grimal, M. Talmant, and S. Naili. Determination of the random anisotropic elasticity layer using transient wave propagation in a fluid-solid multilayer: Model and experiments. The Journal of the Acoustical Society of America, 125, (4), (2009), pp. 2027-2034. doi:10.1121/1.3087428.

[25] V.-H. Nguyen, A. Abdoulatuf, C. Desceliers, and S. Naili. A probabilistic study of reflection and transmission coefficients of random anisotropic elastic plates. Wave Motion, 64, (2016), pp. 103 - 118. doi:10.1016/j.wavemoti.2016.03.007.

[26] V.-H. Nguyen and S. Naili. Simulation of ultrasonic wave propagation in anisotropic poroelastic bone plate using hybrid spectral/finite element method. International Journal for $\mathrm{Nu}$ merical Methods in Biomedical Engineering, 28, (8), (2012), pp. 861-876. doi:10.1002/cnm.2462.

[27] V.-H. Nguyen and S. Naili. Ultrasonic wave propagation in viscoelastic cortical bone plate coupled with fluids: a spectral finite element study. Computer Methods in Biomechanics and Biomedical Engineering, 16, (9), (2013), pp. 861-876. doi:10.1080/10255842.2011.645811.

[28] K. Bathe. Finite Element Procedures. Prentice Hall, (1996). 
[29] C. Soize. Non-gaussian positive-definite matrix-valued random fields for elliptic stochastic partial differential operators. Computer Methods in Applied Mechanics and Engineering, 195, (1-3), (2006), pp. 26-64. doi:10.1016/j.cma.2004.12.014.

[30] E. Jaynes. Information theory and statistical mechanics. The Physical Review, 106, (4), (1957), pp. 620-630. doi:10.1103/physrev.108.171.

[31] E. Jaynes. Information theory and statistical mechanics. II. The Physical Review, 108, (2), (1957), pp. 171-190. doi:10.1103/physrev.108.171.

[32] M. Mehta. Random Matrices. Academic Press, (2004).

[33] C. Soize. Maximum entropy approach for modeling random uncertainties in transient elastodyanmics. Journal of the Acoustical Society of America, 109, (5), (2001), pp. 1979-1996. doi:10.1121/1.1360716.

[34] C. Desceliers, C. Soize, S. Naili, and G. Haiat. Probabilistic model of the human cortical bone with mechanical alterations in ultrasonic range. Mechanical Systems and Signal Processing, 32, (2012), pp. 170 - 177. doi:10.1016/j.ymssp.2012.03.008.

[35] X. Dong and X. Guo. The dependence of transversaly isotropic elasticity of human femoral cortical bone on porosity. Journal of Biomechanics, 37, (2004), pp. 1281-1287. doi:10.1016/j.jbiomech.2003.12.011.

[36] S. C. Cowin. Bone mechanics handbook. CRC Press, Boca Raton, FL, 2nd edition, (2001).

[37] L. Wang, S. Fritton, S. Cowin, and S. Weinbaum. Fluid pressure relaxation depends upon osteonal microstructure: modeling an oscillatory bending experiment. Journal of Biomechanics, 32, (1999), pp. 663-672. doi:10.1016/s0021-9290(99)00059-7.

[38] V.-H. Nguyen, T. Lemaire, and S. Naili. Poroelastic behaviour of cortical bone under harmonic axial loading: A finite element study at the osteonal scale. Medical Engineering $\mathcal{E}$ Physics, 32, (4), (2010), pp. 384 - 390. doi:10.1016/j.medengphy.2010.02.001.

[39] G. Rosi, V.-H. Nguyen, and S. Naili. Reflection of acoustic wave at the interface of a fluidloaded dipolargradient elastic half-space. Mechanics Research Communications, 56, (2014), pp. 98 - 103. doi:10.1016/j.mechrescom.2013.12.002.

[40] G. Rosi, V.-H. Nguyen, and S. Naili. Numerical investigations of ultrasound wave propagating in long bones using a poroelastic model. Mathematics and Mechanics of Solids, 21, (1), (2016), pp. 119-133. doi:10.1177/1081286515582889. 